\title{
Antioxidant and antimicrobial effects of grape pomace extracts
}

\author{
C.E. Luchian ${ }^{1}$, V.V. Cotea ${ }^{1}$, L. Vlase ${ }^{2}$, A.M. Toiu ${ }^{2}$, L.C. Colibaba ${ }^{1}$, I.E. Răschip ${ }^{3}$, G. Nadăş ${ }^{4}$, A.M. Gheldiu ${ }^{2}$ \\ C. Tuchiluş ${ }^{5}$, and L. Rotaru ${ }^{1}$ \\ 1 "Ion Ionescu de la Brad" University of Agricultural Sciences and Veterinary Medicine, Iaşi, Romania \\ 2 "Iuliu Hatieganu" University of Medicine and Pharmacy, Faculty of Pharmacy, Cluj-Napoca, Romania \\ ${ }^{3}$ Romanian Academy, "Petru Poni” Institute of Macromolecular Chemistry, Iaşi, Romania \\ ${ }^{4}$ University of Agricultural Sciences and Veterinary Medicine Cluj-Napoca, Romania \\ 5 "Grigore T. Popa" University of Medicine and Pharmacy, Iaşi, Romania
}

\begin{abstract}
The use of antioxidants and antibacterials in food industry has become increasingly necessary to ensure the high quality of food. Grape pomace is the main by-product of winemaking industry that concentrates bioactive metabolites with more studied antioxidant activity and possible antibacterial activity. The grape pomace contains fragmented skin, broken cells, pulp remains, stalks and seeds with high amount of phenolic compounds due to their poor extraction during the winemaking process. Anthocyanins, catechins, flavonol glycosides, phenolic acids, alcohols and stilbenes have been identified among the compounds present in grape pomace. In this study, antibacterial activity against different pathogens (Escherichia coli ATCC 25922, Candida albicans ATCC 90028, Pseudomonas aeruginosa ATCC 27853 and Staphylococcus aureus ATCC 25923) was evaluated and the relation with polyphenols content and antioxidant activity of grape pomace from selected grapes from Iaşi vineyard was studied. The grape pomace samples were obtained after the fermentation process from the 2017 harvest of Sauvignon Blanc, Traminer, Busuioacă de Bohotin, Cabernet Sauvignon, Merlot, Fetească Neagră and Fetească Regală grape varieties. The antioxidant properties were evaluated using the DPPH method, FRAP assay and Folin Ciocalteu method. The content of resveratrol was quantified using an HPLC method. Samples with antioxidant activity showed the highest phenolics content. This study reveals that grape pomace is a potential source of natural antioxidant agents. The pomace extracts were tested to establish the effects on Gram-positive and Gram-negative bacteria. The analysed samples exhibited insignificant antibacterial activity and the method requires optimization. Grape marc represents an important source of resveratrol and other bioactive compounds that could be a valuable source of antioxidants for further utilization in food and pharmaceutical industry.
\end{abstract}

\section{Introduction}

The main organic waste produced in wine industries is grape pomace, representing $13 \%$ to $25 \%$ of the total weight of processed fruit [1].

Wine-making by-products still contain significant amounts of various bioactive compounds [2]. Grape pomace contain numerous phenolic acids and flavonoids which could possess antioxidant activity and antibacterial activities.

The antioxidant activity of phenolic compounds depends on the number of hydroxyl groups in the molecule and the activity can be strengthened by steric hindrance. The electron withdrawing attribute of the carboxylate group in benzoic acids has a negative effect on the H-donating abilities of the hydroxy benzoates. Hydroxylated cinnamates are more effective than benzoate counterparts [3]. The antioxidant capacity of phenolic substances can perform as free radical scavengers, hydrogen donators, metal chelators and singlet oxygen quenchers.

Antibacterial properties of phenolic substances may be due to iron deprivation or hydrogen bounding with vital proteins, such as microbial enzymes $[3,4]$. Flavonoids are naturally occurring phenolic compounds which include anthoxanthins (flavones, flavonols, flavanones, flavanols, chalcones and isoflavones), anthocyanins, leucoxanthins and flavonoidal alkaloids [5,6]. Grape seeds and skins are often used as a dietary supplement, containing vitamins, minerals, and polyphenols. The polyhenols of grape seeds, such as catechins, epicatechin, procyanidin or some dimmers and trimers, have been recognized for their health benefits. Catechins and their epimers serve as powerful antioxidants for directly eliminating superoxide anion radicals. Proanthocyanidins are apparently responsible for the action on the cardiovascular system [5].

Grape pomace represents a potential source of resveratrol, one of the secondary plant metabolites [7]. This compound has insulin sensitizing effects and maintains the blood glucose homeostasis, with numerous positive influences on health including anticancer action, neuroprotective activity and antiaging properties $[8,9]$.

Since wine-making residues composition depends on grape variety, climate conditions, vineyard location, and winemaking process, it is relevant to study the antioxidant effects and antibacterial activity of each variety separately and so to investigate the biotechnological applications of natural sources [4]. 
Therefore, more antioxidant and antibacterial studies on grape pomace extracts are still needed in order to identify the appropriate mechanisms to evaluate the maximum potential for each grape variety.

The aim of the present study was to evaluate the composition of phenolic compounds in grape pomace from seven different varieties from Iaşi Vineyard and assess their antioxidant and antibacterial activities to determine their potential as source of natural antioxidants and antimicrobials.

\section{Materials and methods}

\subsection{Grapes samples and winemaking}

The necessary plant material for the investigation was represented by Merlot (V1), Cabernet Sauvignon (V2), Busuioacă de Bohotin (V3), Muscat Ottonel (V4), Fetească Neagră (V5), Traminer (V6), Sauvignon Blanc (V7) grape varieties, harvested in autumn of 2017 at full maturity from Iaşi vineyard - Bucium and Bohotin regions. The grapes were crushed and destemed, succeded by maceration phase ( 24 hours for white varieties and 5 days for reds at $10^{\circ} \mathrm{C}$ ) and then pressed. The marc resulted from pressing operation was dried and stored in the freezer until analysis moment. At the moment of the analysis, the marc samples were defrosted and dried in air stream until constant weight, transformed to a powder and extracted with different solvents.

\subsection{Extraction phase}

For the extraction, two different methods were compared. One extraction was realised using a $50 \%$ mixture of water and ethanol. Another extraction was performed with a $50 \%$ mixture of water and methanol. Two series of extracts were prepared by mixing $2 \mathrm{~g}$ of solid samples with $5 \mathrm{~mL}$ of aqueous mixture, followed by stirring for $24 \mathrm{~h}$ at room temperature, and then centrifuged at $4500 \mathrm{rpm}$ for 20 minutes at $4{ }^{\circ} \mathrm{C}$. The supernatants were recovered and used for analysis.

\subsection{Chemicals}

Methanol, ethanol and acetonitrile of HPLC gradient, ammonium acetate, formic acid, acetic acid of analyticalgrade were purchased from Merck (Merck KgaA, Darmstadt, Germany). Trans-resveratrol was purchased from Sigma (Sigma-Aldrich Chemie GmbH, Munich, Germany). Other chemicals used were of biochemistry grade.

\subsection{Methods of analyses}

\subsubsection{Determination of total polyphenolic content}

The total phenolic content (TPC) of the extracts was purchased using the Folin-Ciocalteu assay, with some revisions. The absorbance was measured at $760 \mathrm{~nm}$, using a JASCO UV-VIS spectrophotometer. Standard curve was prepared by using different concentrations of gallic acid. TPC was expressed as $\mathrm{mg}$ gallic acid/ $\mathrm{mL}$ extract (mg GAE/mL extract) [10]. All samples were analysed in triplicate.

\subsubsection{DPPH assay}

The DPPH (2,2-diphenyl-1-picrylhydrazyl) method was performed to assess radical scavenging activity, by bleaching of purple methanolic solution of the stable radical. Hydrophilic and lipophilic synthetic antioxidants, quercetin and butylated hydroxytoluene (BHT) were used as standards reagents. The measure of antioxidant effect is the disappearance of the DPPH absorbtion by the action of antioxidants. $20 \mu \mathrm{l}$ of diluted extracts were added to $980 \mu \mathrm{l}$ DPPH solution $(100 \mu \mathrm{M})$. The reduction in absorbance was measured at $517 \mathrm{~nm}$, using a UV-VIS JASCO V-530 spectrophotometer after 30 minutes of incubation period. After adding individual samples, the percentage inhibition of DPPH radical was calculated according to the formula: $\mathrm{I}=100\left(\mathrm{~A}_{c}-\mathrm{A}_{s}\right) / \mathrm{A}_{c}$, where $\mathrm{I}-\mathrm{DPPH}$ inhibition (\%), $\mathrm{A}_{c}$ - absorbance of control sample, $\mathrm{A}_{s}$ - absorbance of tested sample. Antioxidant activity was also expressed as inhibitory concentration $\mathrm{IC}_{50}$ (calculated graphically) that refers to the concentration of the sample required to cause a 50\% decrease in initial DPPH radical absorbance. Experiments were carried out in triplicate [11-13].

\subsubsection{Ferric Reducing Antioxidant Power assay}

The FRAP (ferric reducing antioxidant power) assay is based on the colour variation of a complex with $\mathrm{Fe}^{+3}$ ion of the 2,4,6-tri(2-pyridyl)-1,3,5-triazine (TPTZ) radical due to the reduction of the ferric ion to the ferrous iron $\left(\mathrm{Fe}^{+2}\right)$ in this complex. Trolox solution was used as reference. A curve absorbance was built according to Trolox mass, the correlation coefficient $\left(\mathrm{R}^{2}\right)$ for this curve being 0.992 . The final results were converted to $\mu \mathrm{M}$ Trolox equivalents/100 mL extract [14]. All samples were analysed in triplicate.

\subsubsection{Antimicrobial susceptibility tests}

The antimicrobial activity was studied using Gram positive bacteria (Staphylococcus aureus ATCC 25923), Gram negative bacteria (Escherichia coli ATCC 25922, Pseudomonas aeruginosa ATCC 27853) and a pathogenic yeasts (Candida albicans ATCC 90028).

Antimicrobial tests of selected microorganisms were carried out using a disc-diffusion method (CLSI). A small amount of each microbial culture was diluted in sterile $0.9 \% \mathrm{NaCl}$ until the turbidity was equivalent to McFarland standard no. 0.5 (106 CFU/mL). Mueller Hinton agar (Oxoid) and Mueller-Hinton agar Fungi (Biolab) were inoculated with the suspensions of the tested microorganisms. Sterile stainless steel cylinders $(5 \mathrm{~mm}$ internal diameter; $10 \mathrm{~mm}$ height) were applied on the agar surface in Petri plates. Then, $100 \mu \mathrm{L}$ of the tested compounds (1-7), with a concentration of $10 \mathrm{mg} / \mathrm{mL}$ each were added into cylinders. The plates were left 10 minutes at room temperature to ensure the equal diffusion of the compound in the medium and then incubated at $35^{\circ} \mathrm{C}$ for 24 hrs.

As reference there were used commercial available discs with antimicrobial drugs containing Ciprofloxacin $(5 \mu \mathrm{g} / \mathrm{disk})$, Fluconazole $(25 \mu \mathrm{g} / \mathrm{disk})$ and Voriconazole $(1 \mu \mathrm{g} /$ disk $)$. After incubation, the diameters of inhibition were measured. All assays were carried out in triplicate $[15,16]$. 


\subsubsection{Determination of resveratrol using HPLC}

A methanolic solution $(10 \mathrm{mg} / \mathrm{mL})$ of trans-resveratrol was prepared and stored at $4{ }^{\circ} \mathrm{C}$ and protected from light. Before analysis, aliquots of the prepared solution were adequately diluted with bidistilled water. Cis-resveratrol was prepared from a standard solution of trans-resveratrol after its irradiation for 10 minutes using an UV lamp $(254 \mathrm{~nm})$.

Sample preparation. The samples were diluted ten folds with bidistilled water, then centrifuged at $10,000 \mathrm{rpm}$ for 5 minutes. An aliquot was then injected into the chromatographic system.

The experiment was performed using an Agilent 1100 HPLC Series system (Agilent, SUA) equipped with an autosampler G1311A. For the separation, a reversed-phase Zorbax SB-C18 analytical column $(100 \times 3.0 \mathrm{~mm}$ i.d., $3.5 \mu \mathrm{m}$ particles) was used. The column was operated at $40^{\circ} \mathrm{C}$ in a G1316A oven. For the elution a degasser (G1322A), and a binary gradient pump (G1311A) were used. The isocratic elution was achieved using a mixture of $1 \mathrm{mM}$ ammonium acetate/acetonitrile (73/27, v/v). The flow rate was $1 \mathrm{~mL} / \mathrm{min}$ and the injection volume $5 \mu \mathrm{L}$. All solvents were filtered using $0.5 \mathrm{~mm}$ (Sartorius) filters and degassed in an ultrasonic bath. The detection of transand cis-resveratrol was carried out using an Agilent Ion Trap VL mass spectrometer (Agilent, SUA). The mass spectrometer was operated using an atmospheric pressure chemical ionisation (APCI) ion source in negative mode. The nitrogen was used as nebulising and dry gas. The APCI heater was set at $350{ }^{\circ} \mathrm{C}$, the nebulizer pressure $60 \mathrm{psi}$, dry gas flow was $5 \mathrm{~L} / \mathrm{min}$ and was heated at $250^{\circ} \mathrm{C}$. The mass spectrometer operated in multiple reactions monitoring mode and was set to monitor the transition $\mathrm{m} / \mathrm{z}$ $227 \rightarrow \mathrm{m} / \mathrm{z} 185$.

Chromatographic and mass spectrometric data acquisition were performed using Chemstation software (Agilent Technologies, Palo Alto, CA, USA), version B.01.03 and LC/MSD Trap Control (Bruker Daltonik, GmbH, Bremen, Germany), version 5.3, while data processing was performed using LC/MSD Data Analysis and Quant Analysis software (Bruker Daltonik, GmbH, Bremen, Germany), version 1.7. In order to find the optimum ion source (MS interface) and mobile phase composition the signal to noise $(\mathrm{S} / \mathrm{N})$ ratios were calculated for each injection [17].

\section{Results and discussion}

\subsection{Polyphenols analysis}

Total phenolic content varied considering the analysed extracts. Different values of polyphenols concentration were obtained depending on the solvent used for the extraction.

The obtained concentrations of total polyphenols from analysed samples are presented in Table 1 .

In the case of the extract obtained with ethanol, the higher concentration in total polyphenols was registered in V5 extract ( $2.77 \mathrm{mg} \mathrm{GAE} / \mathrm{mL})$, V4 (2.76 $\mathrm{mg} \mathrm{GAE} / \mathrm{mL})$, and V1 $(2.70 \mathrm{mg} \mathrm{GAE} / \mathrm{mL})$, followed by V6 extract (2.57 mg GAE/mL). Smaller contents of polyphenols were found in samples V3, V2 and V7. On the other hand, in the case of the methanol extract, the highest values for the analysed parameters were recorded at V1 $(2.42 \mathrm{GAE} / \mathrm{mL})$,
Table 1. TPC in analysed extracts $( \pm \mathrm{SD})$.

\begin{tabular}{|c|c|c|}
\hline Sample & $\begin{array}{c}\text { TPC (mg GAE/mL) } \pm \text { SD } \\
\text { Extract with ethanol }\end{array}$ & $\begin{array}{c}\text { TPC }(\mathbf{m g} \text { GAE/mL) } \pm \text { SD. } \\
\text { Extract with methanol }\end{array}$ \\
\hline V1 & $2.7075 \pm 0.002$ & $2.421 \pm 0.09$ \\
\hline V2 & $2.08 \pm 0.002$ & $0.642 \pm 0.03$ \\
\hline V3 & $2.1375 \pm 0.001$ & $0.404 \pm 0.02$ \\
\hline V4 & $2.76 \pm 0.001$ & $0.771 \pm 0.05$ \\
\hline V5 & $2.7775 \pm 0.002$ & $1.452 \pm 0.07$ \\
\hline V6 & $2.5725 \pm 0.003$ & $1.242 \pm 0.05$ \\
\hline V7 & $2.03 \pm 0,003$ & $0.884 \pm 0.06$ \\
\hline
\end{tabular}

\pm SD standard deviation; GAE - gallic acid.

Table 2. Antioxidant activity of extracts using DPPH method.

\begin{tabular}{|c|c|c|}
\hline Sample & $\begin{array}{c}\mathbf{I C}_{\mathbf{5 0}}(\boldsymbol{\mu g} / \mathbf{m L}) \\
\text { Extract with ethanol }\end{array}$ & $\begin{array}{c}\mathbf{I C}_{\mathbf{5 0}}(\boldsymbol{\mu g} / \mathbf{m L}) \\
\text { Extract with methanol }\end{array}$ \\
\hline V1 & $24.21 \pm 0.82$ & $98.142 \pm 4.31$ \\
\hline V2 & $34.60 \pm 1.02$ & $134.48 \pm 5.27$ \\
\hline V3 & $33.42 \pm 0.91$ & $144.9 \pm 6.41$ \\
\hline V4 & $20.93 \pm 0.79$ & $132.17 \pm 5.11$ \\
\hline V5 & $20.59 \pm 0.75$ & $115.78 \pm 4.29$ \\
\hline V6 & $30.71 \pm 0.96$ & $119.86 \pm 4.35$ \\
\hline V7 & $44.04 \pm 1.24$ & $127.96 \pm 4.74$ \\
\hline Quercetin & $5.59 \pm 0.13$ & $5.59 \pm 0.13$ \\
\hline BHT & $15.88 \pm 1.06$ & $15.88 \pm 1.06$ \\
\hline
\end{tabular}

Note: Values are the mean $\pm \operatorname{SD}(n=3)$.

Table 3. Antioxidant activity of extracts using FRAP method.

\begin{tabular}{|l|c|c|}
\hline Sample & $\begin{array}{c}\boldsymbol{\mu M} \text { Trolox } / \mathbf{1 0 0} \mathbf{~ m L} \\
\text { Extract with ethanol }\end{array}$ & $\begin{array}{c}\boldsymbol{\mu} \text { M Trolox } / \mathbf{1 0 0 ~} \mathbf{~ L L} \\
\text { Extract with methanol }\end{array}$ \\
\hline V1 & $1432.37 \pm 9.68$ & $1164.22 \pm 12.46$ \\
\hline V2 & $957.40 \pm 8.25$ & $359.75 \pm 6.84$ \\
\hline V3 & $1003.39 \pm 9.01$ & $186.09 \pm 5.58$ \\
\hline V4 & $1435.27 \pm 10.37$ & $406.17 \pm 7.53$ \\
\hline V5 & $1688.51 \pm 11.43$ & $782.09 \pm 9.31$ \\
\hline V6 & $1252.91 \pm 10.05$ & $473.73 \pm 8.27$ \\
\hline V7 & $937.09 \pm 9.29$ & $434.35 \pm 8.01$ \\
\hline Trolox & $2073.91 \pm 26.08$ & $2073.91 \pm 26.08$ \\
\hline
\end{tabular}

Note: Values are the mean $\pm \mathrm{SD}(n=3)$.

V5 $(1.45 \mathrm{GAE} / \mathrm{mL})$ and V6 $(1.24 \mathrm{GAE} / \mathrm{mL})$, followed by $\mathrm{V} 7(1.24 \mathrm{GAE} / \mathrm{mL})$ and $\mathrm{V} 4$ extracts $(0.88 \mathrm{GAE} / \mathrm{mL})$.

The extraction method in which ethanol was used was more efficient in determining the amount of phenolic.

\subsection{Antioxidant activity assay by DPPH method}

In order to evaluate the ability of extracts and synthetic antioxidants quercetin and BHT to donate the hydrogen atom, the stable free radical DPPH was used. The results obtained for the evaluation of the antioxidant activity using the DPPH bleaching assay are presented in Table 2. All extracts were able to reduce DPPH radical with different degrees of scavenging activity. 
Table 4. Antibacterial and Antifungal Activities of the Tested Compounds.

\begin{tabular}{|c|c|c|c|c|}
\hline \multirow{2}{*}{ Compounds } & \multicolumn{4}{|c|}{ Diameter of inhibition zones (mm) } \\
\cline { 2 - 5 } & $\begin{array}{c}\text { S. aureus } \\
\text { ATCC 25923 }\end{array}$ & $\begin{array}{c}\text { E. coli } \\
\text { ATCC 25922 }\end{array}$ & $\begin{array}{c}\text { Pseudomonas aeruginosa } \\
\text { ATCC 27853 }\end{array}$ & $\begin{array}{c}\text { C. albicans } \\
\text { ATCC 90028 }\end{array}$ \\
\hline 1 & - & - & - & $12.0 \pm 0.00$ \\
\hline 2 & - & - & - & - \\
\hline 3 & - & - & - & - \\
\hline 4 & - & - & - & - \\
\hline 5 & - & - & - & - \\
\hline 6 & - & - & - & $* \mathrm{NT}$ \\
\hline 7 & - & - & - & $30.0 \pm 0.00$ \\
\hline Ciprofloxacin $5 \mu \mathrm{g} / \mathrm{disc})$ & $27.7 \pm 0.06$ & $30.0 \pm 0.00$ & $30.0 \pm 0.00$ & $29.5 \pm 0.50$ \\
\hline Fluconazol $(25 \mu \mathrm{g} / \mathrm{disc})$ & $\mathrm{NT}^{*}$ & $\mathrm{NT}^{*}$ & $\mathrm{NT}^{*}$ & $\mathrm{NT}$ \\
\hline Voriconazol $(1 \mu \mathrm{g} / \mathrm{disc})$ & $\mathrm{NT}^{*}$ & $\mathrm{NT}^{*}$ & & \\
\hline
\end{tabular}

*NT-not tested.

A lower $\mathrm{IC}_{50}$ value represents a higher bleaching effect, thus a better antioxidant activity. Differences can be observed depending on the solvent used in the extraction.

All analysed extracts showed lower DPPH scavenging activity than standards quercetin and BHT. In the case of extract obtained with ethanol, the highest radical scavenging activity was determined for extract V5 and V4 $(20.59 \mu \mathrm{g} / \mathrm{mL}$, and $20.93 \mu \mathrm{g} / \mathrm{mL}$ respectively), with positive correlation between scavenging activity on DPPH and total phenolic content. The extracts V2 and V3 showed comparable antioxidant activity by DPPH method, and the results could also be correlated with the content in total polyphenols. The lower antioxidant effect was observed for extracts V3, V2, V7 ( $\mathrm{IC}_{50}$ value $=33.42 \mu \mathrm{g} / \mathrm{mL}$, $34.60 \mu \mathrm{g} / \mathrm{mL}, 44.04 \mu \mathrm{g} / \mathrm{mL}$ and respectively), and this could be due to the smaller concentration of polyphenols (2.03-2.13 mg GAE/mL). Considering the obtained results by DPPH method, the following order in antioxidant activity could be established: V7 $<\mathrm{V} 2<$ $\mathrm{V} 3<\mathrm{V} 6<\mathrm{V} 1<\mathrm{V} 4<\mathrm{V} 5<\mathrm{BHT}<$ quercetin. According to this method, all analysed extracts showed high antioxidant effects $\left(\mathrm{IC}_{50}<50 \mu \mathrm{g} / \mathrm{mL}\right)$ [12]. For the second extract (with methanol), the strongest antioxidant activity was noted in V1 sample, (with $\mathrm{IC}_{50}=98.142 \mu \mathrm{g} / \mathrm{mL}$ ) and V5 $(115.78 \mu \mathrm{g} / \mathrm{mL})$ extract, with positive correlation between scavenging activity on DPPH and total phenolic content. Considering the obtained results, the following order in antioxidant activity could be established: V3 $<$ V $2<$ $\mathrm{V} 4<\mathrm{V} 7<\mathrm{V} 6<\mathrm{V} 5<\mathrm{V} 1<\mathrm{BHT}<$ quercetin. According to this method, the analysed extracts showed weak antioxidant effects [11].

The results could be related to the presence of higher amounts of phenolic compounds and indicates that different active principles can contribute to antioxidant properties of natural products. The various antioxidant properties of these extracts may be due to the variability of composition and content in phytochemicals, and the method used for extraction. Ethanol solvent may be preferable for the extraction preparation.

\subsection{Ferric Reducing Antioxidant Power (FRAP) assay}

Table 3 express the evaluation of the antioxidant activity using the FRAP assay.
A positive correlation between scavenging activity determined by FRAP method and total phenolic content was observed. The results are varying in correlation to the used method.

The highest antioxidant activity for the extractions with ethanol was determined for V5 sample and V4 $(1688.51 \mu \mathrm{M}$ Trolox/100 mL, and $1435.27 \mu \mathrm{M}$ Trolox/100 mL respectively). Using the FRAP assay, the following order in antioxidant activity was found: $\mathrm{V} 7<\mathrm{V} 2<\mathrm{V} 3<\mathrm{V} 6<\mathrm{V} 1<\mathrm{V} 4<\mathrm{V} 5<$ Trolox.

In the second situation (extract with methanol), the highest scavenging activity was noted for V1 and V5 extracts $(1164.22 \mu \mathrm{M}$ Trolox $/ 100 \mathrm{~mL}$, and $782.09 \mu \mathrm{M}$ Trolox $/ 100 \mathrm{~mL}$ respectively). Using the FRAP assay, the following order in antioxidant activity was found: V3 $<$ V2 $<\mathrm{V} 4<\mathrm{V} 7<\mathrm{V} 6<\mathrm{V} 5<\mathrm{V} 1<$ Trolox.

The antioxidant activity using the FRAP assay could be correlated with the results obtained by DPPH assay.

\subsection{Antimicrobial activity}

The diameters of the inhibition zones (in $\mathrm{mm}$ ) corresponding to the tested compounds are shown in Table 1. All assays were carried out in triplicate. Results are expressed as means \pm SD. Against Gram negative and Gram negative bacteria all the tested samples have insignificant activity. The V1 and V7 extracts registered a reduced antifungal activity. The influence of grape pomace extract on the growth of fungi and bacteria is expressed in Fig. 1.

\subsection{Determination of resveratrol using HPLC}

Resveratrol is a very polar compound and it exist as trans- and cis- isomers, both present in grapes [18]. For the extraction of resveratrol, the yield was determinate by using ethanol. The resveratrol content identified using a chromatographic determination is presented in Table 5.

Figure 2 express a chromatogram of trans- and cis-resveratrol standard solution at LOQ (limit of quantification level). The concentration of trans-resveratrol in grape pomace extract varied considerably, depending on the grape variety. 


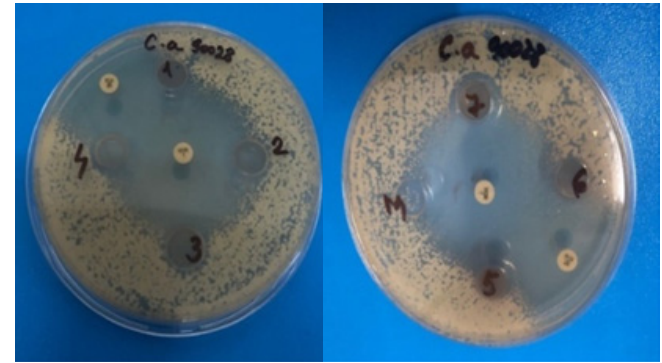

A

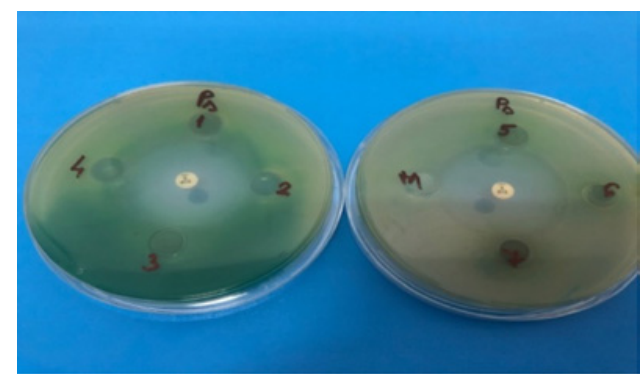

C

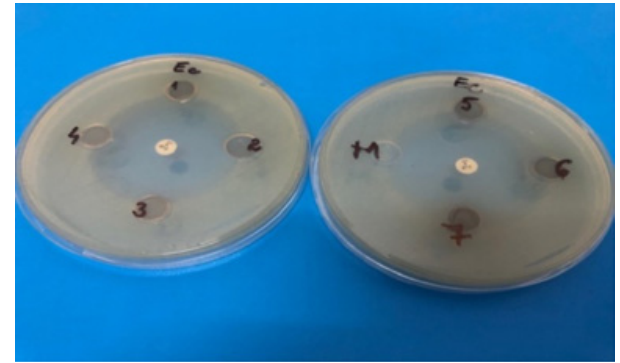

B

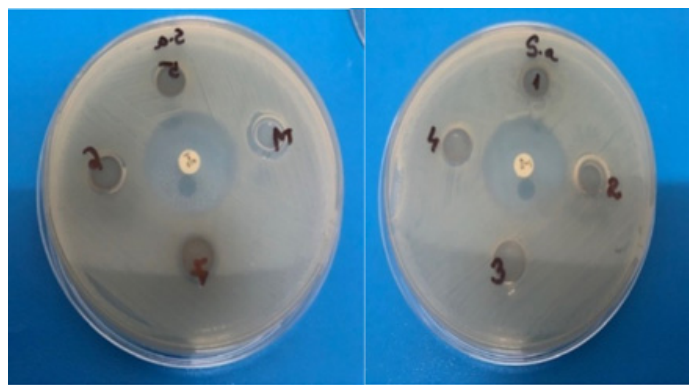

D

Figure 1. The influence of grape pomace extract on the growth of Candida albicans ATCC 90028 (A), Escherichia coli ATCC 25922 (B) and Pseudomonas aeruginosa ATCC 27853 (C), Staphylococcus aureus ATCC 25923 (D).

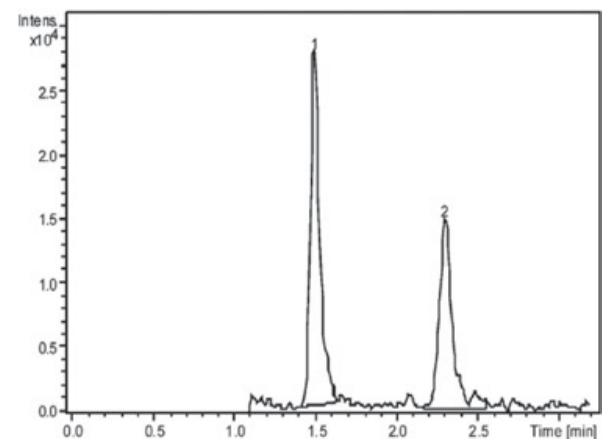

Figure 2. Chromatogram corresponding to a standard mixture of trans- and cis-resveratrol at the limit of quantification level (peak $1=$ trans-resveratrol; peak 2 = cis-resveratrol).

Table 5. The Resveratrol content of pomace extracts.

\begin{tabular}{|c|c|c|c|c|}
\hline \multirow{2}{*}{ Sample } & \multicolumn{2}{|c|}{$\mu \mathrm{g} / \mathrm{mL}$ conc in extract } & \multirow{2}{*}{$\begin{array}{c}\text { trans }+ \text { cis } \\
\mu \mathrm{g} / \mathrm{mL}\end{array}$} & \multirow{2}{*}{$\begin{array}{c}\operatorname{trans} / c i s \\
\mu \mathrm{g} / \mathrm{mL}\end{array}$} \\
\hline & conc trans & conc cis & & \\
\hline V1 & $23.54 \pm 0.12$ & $2.01 \pm 0.06$ & $25.55 \pm 0.09$ & $11.72 \pm 0.12$ \\
\hline $\mathrm{V} 2$ & $13.21 \pm 0.15$ & $3.27 \pm 0.11$ & $16.48 \pm 0.08$ & $4.04 \pm 0.11$ \\
\hline $\mathrm{V} 3$ & $10.27 \pm 0.13$ & 0 & $10.27 \pm 0.11$ & 0 \\
\hline V4 & $22.68 \pm 0.08$ & 0 & $22.68 \pm 0.13$ & 0 \\
\hline V5 & $15.49 \pm 0.10$ & 0 & $15.49 \pm 0.09$ & 0 \\
\hline V6 & $15.33 \pm 0.12$ & 0 & $15.33 \pm 0.10$ & 0 \\
\hline V7 & $1.43 \pm 0.09$ & 0 & $1.43 \pm 0.15$ & 0 \\
\hline
\end{tabular}

Each value is the mean $\pm \mathrm{SD}$ of three independent measurements.

The content of trans-resveratrol in grape pomace extracts was relatively high, ranging from $1.43 \mu \mathrm{g} / \mathrm{mL}$ (V7) and $23.54 \mu \mathrm{g} / \mathrm{mL}(\mathrm{V} 1)$. The cis-resveratrol concentration was very low or below the limit of detection.

\section{Conclusions}

The results showed a close relationship between content of phenolic compounds and antioxidant capacity. All extracts were able to reduce DPPH radical with different degrees of antioxidant activity.

A positive correlation between scavenging activity determined by FRAP method and total phenolic content being observed. Extract with ethanol showed better results for determination of phenolic compounds from grape pomace. The analysed grape varieties showed a good potential as source of bioactive compounds that could be applied as natural antioxidants in food and cosmetic products, in order the increase their shelf life. Against Gram negative and Gram positive bacteria all the tested samples have an insignificant activity. The V1 and V7 extracts registered a reduced antifungal activity. Grape marc represents an important source of transresveratrol, with great potential for application in food and pharmaceutical industry.

The research was funded by the PN-III-P1-1.1-TE-2016-2038 project, no. 77/8.05.2018 and FDI project - final registration code CNFIS-FDI-2019-0267.

\section{References}

[1] M. Corrales, A. Fernandez, M.G. Vizoso, P. Butz, C. Franz, E. Schuele, B. Tausche, Food Chem. Toxicol. 48, 3471 (2010)

[2] A. Frum, C. Georgescu, F.G. Gligor, E. Lengyel, D.I. Stegarus, C.M. Dobrea, O. Tita, St. Cerc. St. CICBIA 19, 45 (2018)

[3] F. Kabir, M.S. Sultana, H. Kurnianta, Afr. J. Biotechnol. 14, 2157 (2015)

[4] L. Sanhueza, M. Tello, M. Vivanco, L. Mendoza, M.Wilkens, J. Adv. Microbiol. 4, 225 (2014) 
[5] P. Maisuthisakul, Braz. Arch. Biol. Technol. 54, 2 (2011)

[6] G. Spigno, D.M. De Faveri, J. Food Eng. 78, 793 (2005)

[7] N. Păun, V. Niculescu, UASVM Agric. J. 68, 2 (2011)

[8] E. Anderson, The effects of Estrogen, Grape Pomace, and Resveratrol Supplementation on Glucoze Tolerance and Insulin Signaling in Ovariectomized Rats (2017)

[9] C.Y. Ranjitha, S. Priyanka, R. Deepika, G.P. Smitha Rani, J. Sahana, T.R. Prashith Kekud, WJPPS 3, 1483 (2014)

[10] H.P.S. Makkar, M. Bluemmel, N.K. Borowy, K. Becker, J. Sci. Food Agric. 61, 161 (1993)

[11] A. Toiu, A. Mocan, L. Vlase, A.E. Pârvu, D.C. Vodnar, A.M. Gheldiu, C. Moldovan, I. Oniga, Front. Pharmacol. 2, 9 (2018)

[12] J.H. Andriamadio, L.H. Rasoanaivo, D. Benedec, L. Vlase, A.M. Gheldiu, M. Duma, Nat. Prod. 29, 2188 (2015)

[13] C.E. Pop, M. Pârvu, A. Arsene, A.E. Pârvu, D.C. Vodnar, M. Tarcea, A.M. Toiu, L. Vlase, Farmacia J. 4, 65 (2017)
[14] K. Thaipong, U. Boonprakob, K. Crosby, L. Cisneros-Zevallos, D. Hawkins Byrne, J. Food Compos. Anal. 19, 669 (2006)

[15] J.H. Rex, M.A. Ghannoum, B.D. Alexander, D. Andes, S.D. Brown, D.J. Diekema, A. Espinel-Ingroff, C.L. Fowler, E.M. Johnson, C.C Knapp, M.R. Motyl, L. Ostrosky-Zeichner, M.A. Pfaller, D.J. Sheehan, T.J. Walsh, CLSI 24, (2009)

[16] M.P. Weistein, J.B. Patel, A.M. Bobenchik, S. Campeau, S. Cullen, M. Galas, H. Gold, R. Humphries, T.J. Kirn, J.S. Lewis, B. Limbago, A.J. Mathers, T. Mazzulli, S.S. Richter, M. Satlin, A.N. Schuetz, J.M. Swenson, P.D. Tamma, CLSI 29, (2019)

[17] L. Vlase, B. Kiss, S.E. Leucuta, S. Gocan, J. Liq. Chromatogr. Relat. Technol. 32, 2105 (2009)

[18] L. Cai, J.A. Koziel, M. Dharmadhikari, J. van Leeuwen, J. Chromatogr. 1216, 281 (2009)

[19] J.G. Prado Martin, E. Porto, C.B. Correa, S.M. de Alencar, E. Micotti di Gliria, I.S. Ribeiro, J. Nat. Prod. 5, 27 (2012) 